Review Article

\title{
Impact of Statins on Cognitive Deficits in Adult Male Rodents after Traumatic Brain Injury: A Systematic Review
}

\author{
Weijun Peng, ${ }^{1,2}$ Jingjing Yang, ${ }^{1}$ Bo Yang, ${ }^{1,2}$ Lexing Wang, \\ Xin-gui Xiong, ${ }^{1,2}$ and Qinghua Liang ${ }^{1,2}$ \\ ${ }^{1}$ Institute of Integrated Medicine, Xiangya Hospital, Central South University, No. 87 Xiangya Road, Changsha, \\ Hunan 410008, China \\ ${ }^{2}$ Key Laboratory of Chinese Gan of State Administration of Traditional Chinese Medicine, Changsha 410008, China \\ ${ }^{3}$ Institute of Integrated Medicine, Hunan Cancer Hospital, Changsha 410013, China \\ ${ }^{4}$ The Affiliated Cancer Hospital of Xiangya School of Medicine, Central South University, Changsha 410013, China
}

Correspondence should be addressed to Bo Yang; yangbo0503@gmail.com

Received 21 May 2014; Accepted 12 July 2014; Published 23 July 2014

Academic Editor: Konstantinos P. Economopoulos

Copyright (C) 2014 Weijun Peng et al. This is an open access article distributed under the Creative Commons Attribution License, which permits unrestricted use, distribution, and reproduction in any medium, provided the original work is properly cited.

The efficacy of statin treatment on cognitive decline is controversial, and the effect of statins on cognitive deficits in individuals with traumatic brain injury (TBI) has yet to be investigated. Therefore, we systematically reviewed the effect of statins on cognitive deficits in adult male rodents after TBI. After identifying eligible studies by searching four electronic databases on February 28, 2014, we assessed study quality, evaluated the efficacy of statin treatment, and performed stratified metaregression and metaregression to assess the influence of study design on statin efficacy. Eleven studies fulfilled our inclusion criteria from a total of 183 publications. The overall methodological quality of these studies was poor. Meta-analysis showed that statins exert statistically significant positive effects on cognitive performance after TBI. Stratified analysis showed that atorvastatin has the greatest effect on acquisition memory, simvastatin has the greatest effect on retention memory, and statin effects on acquisition memory are higher in closed head injury models. Metaregression analysis further showed that that animal species, study quality, and anesthetic agent impact statin effects on retention memory. We conclude that statins might reduce cognitive deficits after TBI. However, additional well-designed and well-reported animal studies are needed to inform further clinical study.

\section{Introduction}

Traumatic brain injury (TBI) is a leading cause of death and disability in industrialized countries and is the leading cause of long-term disability in children and young adults worldwide [1]. One of the most significant disabilities associated with TBI is short- and long-term cognitive deficits [2]. Approximately $65 \%$ of patients with moderate to severe TBI report long-term problems with cognitive functioning, and as many as $15 \%$ with mild TBI have persistent problems that often include cognitive deficits $[3,4]$. These deficits interfere with work, relationships, leisure, and daily living activities, exacting a personal and economic cost that is difficult to quantify [4]. However, despite substantial efforts, few therapeutic options exist to prevent or alleviate cognitive dysfunction after TBI in humans $[5,6]$.

Statins (3-hydroxy-3-methylglutaryl-coenzyme A reductase inhibitors) are implicated in stroke, Alzheimer's disease, and multiple sclerosis [7] and constitute potential treatment options for TBI due to their pleiotropicity [8]. In experimental TBI, simvastatin increases neurogenesis and suppresses apoptosis $[9,10]$, ameliorates secondary brain damage [11], and attenuates microglial and astroglial activation [12]. Both simvastatin and atorvastatin increase neurogenesis and inhibit neuronal death [13], and atorvastatin also reduces brain edema [14]. Lovastatin improves histological outcome and reduces inflammation [15]. Furthermore, simvastatin, lovastatin, and atorvastatin restore cognitive deficits caused 
TABLE 1: Key search terms used in database searches.

\begin{tabular}{lll}
\hline Traumatic brain injury & Statins & Compactin \\
\hline Traumatic brain injury & Statins & Mevinolin \\
Traumatic brain injuries & Statin & Rosuvastatin \\
TBI & Atorvastatin & Simvastatin \\
Head injury & Dalvastatin & Pitavastatin \\
Head injuries & Fluvastatin & Pravastatin \\
Brain injury & Lovastatin & Mevastatin \\
Brain injuries & HMG-CoA reductase inhibitors & \\
Injury brain & Hydroxymethylglutaryl-CoA reductase inhibitors & \\
Injuries brain & Hydroxymethylglutaryl-coenzyme A inhibitors & \\
Head trauma & Hydroxymethylglutaryl-CoA inhibitors & \\
\hline
\end{tabular}

TABLE 2: Criteria for study inclusion/exclusion.

\begin{tabular}{|c|c|}
\hline Inclusion criteria & Exclusion criteria \\
\hline (1) Statins were administered. & (1) Statins were not administered. \\
\hline (2) Experimental TBI was induced in rodents. & (2) No control group was used. \\
\hline (3) Cognitive function was measured by the MWM. & $\begin{array}{l}\text { (3) Nonimpact (e.g., cortical ablation) or penetrating (e.g., } \\
\text { missile-induced) TBI was performed. }\end{array}$ \\
\hline (4) Male rodents (i.e., rats or mice) were used. & $\begin{array}{l}\text { (4) Treatment group was administered another neuroprotective } \\
\text { agent in addition to a statin. }\end{array}$ \\
\hline (5) Article was published in English or Chinese language. & $\begin{array}{l}\text { (5) Other types of animals (e.g., sheep, cats, and dogs) were } \\
\text { used. }\end{array}$ \\
\hline \multirow[t]{3}{*}{$\begin{array}{l}\text { (6) A TBI treatment group was treated with a pharmacological agent, } \\
\text { and a control group was administered a placebo after injury. }\end{array}$} & $\begin{array}{l}\text { (6) Only biochemical or physiological outcomes of treatment } \\
\text { efficacy were assessed. }\end{array}$ \\
\hline & (7) Samples included female rodents. \\
\hline & (8) Duplicate publications. \\
\hline
\end{tabular}

by TBI $[13,16]$. However, there is no systematic evidence available that statins improve cognition in humans with TBI. Moreover, as with all drugs, statins can exert undesirable effects. In 2012, the U.S. Food and Drug Administration issued a statement on cognitive impairment as a potential adverse effect of statins [17], with myopathy being the most well-characterized adverse sequelae [18]. Also, longitudinal studies (both randomized trials and observational studies) of the effects of statins on cognition in individuals without dementia have yielded negative results [19-21].

Although statins show promise for treating cognitive impairment caused by TBI, further clinical trials are needed. Furthermore, given the controversy regarding the effect of statins on cognition, a robust and systematic summary of existing data may assist in the design of clinical trials. Therefore, we investigated the efficacy of statins in treating cognitive deficits in experimental animal models of TBI and explored the impact of study design and quality on reported outcome.

\section{Materials and Methods}

2.1. Search Strategy and Study Selection. We searched four electronic databases (PubMed, Medline, Ovid, and ScienceDirect; February 28, 2014) for studies that examined pharmacological treatments for cognitive, behavioral, and motor problems in rodents after TBI. The key search terms (Table 1) were kept broad to capture all potentially relevant articles. Reference lists from the resulting research articles and reviews were used to identify further relevant publications.

To be included in this meta-analysis, a study had to meet several inclusion criteria (Table 2). Three investigators assessed titles and abstracts and obtained copies of articles that described controlled studies of statins in animal models of TBI to determine their eligibility for inclusion. Disagreements among investigators were resolved by consensus after discussion.

2.2. Data Extraction. Two investigators extracted information about the studies including animal species, sample size, type of TBI model, main experimental groups, substances used as experimental and control treatments, method/dose/timing of statin administration, type of anesthetic agent, and time of outcome assessment. Disagreements between investigators were resolved by consensus after discussion.

The Morris water maze (MWM) was used to assess cognition. When cognition was assessed at different times after TBI, only the last day was considered. Cumulative statin 
TABLE 3: The CAMARADES quality items.

\begin{tabular}{|c|c|c|c|c|c|c|c|c|c|c|c|}
\hline Author & (1) & $(2)$ & (3) & (4) & (5) & (6) & (7) & (8) & (9) & $(10)$ & Quality score \\
\hline Abrahamson et al., 2009 [24] & $\sqrt{ }$ & $\sqrt{ }$ & & & $\sqrt{ }$ & & $\sqrt{ }$ & & & $\sqrt{ }$ & 5 \\
\hline Chauhan and Gatto, 2011 [16] & $\sqrt{ }$ & & & & & & $\sqrt{ }$ & & & $\sqrt{ }$ & 3 \\
\hline Indraswari et al., 2012 [25] & $\sqrt{ }$ & $\sqrt{ }$ & $\sqrt{ }$ & $\sqrt{ }$ & $\sqrt{ }$ & & $\sqrt{ }$ & & $\sqrt{ }$ & $\sqrt{ }$ & 8 \\
\hline Lu et al., 2004 [26] & $\sqrt{ }$ & $\sqrt{ }$ & & $\sqrt{ }$ & $\sqrt{ }$ & & $\sqrt{ }$ & $\sqrt{ }$ & & $\sqrt{ }$ & 7 \\
\hline Lu et al., 2007 [13] & $\sqrt{ }$ & $\sqrt{ }$ & & $\sqrt{ }$ & $\sqrt{ }$ & & $\sqrt{ }$ & $\sqrt{ }$ & & $\sqrt{ }$ & 7 \\
\hline Wang et al., 2012 [27] & $\sqrt{ }$ & $\sqrt{ }$ & & & & & $\sqrt{ }$ & $\sqrt{ }$ & & $\sqrt{ }$ & 5 \\
\hline Wang et al., 2007 [28] & $\sqrt{ }$ & $\sqrt{ }$ & & & $\sqrt{ }$ & & & & & $\sqrt{ }$ & 4 \\
\hline Wu et al., 2008 [10] & $\sqrt{ }$ & $\sqrt{ }$ & & $\sqrt{ }$ & $\sqrt{ }$ & & $\sqrt{ }$ & $\sqrt{ }$ & & $\sqrt{ }$ & 7 \\
\hline Jin et al., 2013 [29] & $\sqrt{ }$ & $\sqrt{ }$ & & & & & & $\sqrt{ }$ & & $\sqrt{ }$ & 4 \\
\hline Liu, 2009 [30] & $\sqrt{ }$ & $\sqrt{ }$ & & & & & $\sqrt{ }$ & $\sqrt{ }$ & & $\sqrt{ }$ & 5 \\
\hline Zhang et al., 2012 [31] & $\sqrt{ }$ & $\sqrt{ }$ & & $\sqrt{ }$ & & & & $\sqrt{ }$ & & $\sqrt{ }$ & 5 \\
\hline
\end{tabular}

Note: (1) peer reviewed publication; (2) presence of randomization of subjects into treatment groups; (3) assessment of dose-response relationship; (4) blinded assessment of behavioural outcome; (5) monitoring of physiological parameters such as body temperature; (6) calculation of necessary sample size to achieve sufficient power; (7) statement of compliance with animal welfare regulations; (8) avoidance of anaesthetic agents with marked intrinsic neuroprotective properties (e.g., ketamine); (9) statement of potential conflict of interests; (10) use of a suitable animal model.

dose was taken into consideration when comparing neurobehavioral outcomes among studies.

In cases of missing data, we contacted the authors and requested the additional information. If data were expressed only graphically, numerical values were requested from the authors; if a response was not received, digital ruler software was used to estimate numerical values from the graphs. If required data were not presented or obtainable, the study was excluded from analysis.

2.3. Methodological Quality of Studies. The methodological quality of individual studies was assessed based on a checklist modified from the Collaborative Approach to Meta-Analysis and Review of Animal Data from Experimental Studies (CAMARADES) as previously described with minor modification [22, 23]. The checklist was comprised of 10 items: (1) peer reviewed publication; (2) presence of randomization of subjects into treatment groups; (3) assessment of doseresponse relationship; (4) blinded assessment of behavioural outcome; (5) monitoring of physiological parameters such as body temperature; (6) calculation of necessary sample size to achieve sufficient power; (7) statement of compliance with animal welfare regulations; (8) avoidance of anaesthetic agents with marked intrinsic neuroprotective properties (e.g., ketamine); (9) statement of potential conflicts of interest; (10) use of a suitable animal model. One point was given for evidence of each quality criterion (Table 3 ).

2.4. Statistical Analysis. In line with the Cochrane Handbook for Systematic Reviews of Interventions, the global estimated effect of statin treatment on cognitive outcome was determined by calculating standardized mean difference (SMD; equal to the difference in mean outcome between groups divided by the standard deviation of outcomes among participants, reported in units of standard deviation) and 95\% confidence intervals (CI) using a random effects model to avoid heterogeneity [23]. SMD is used as a summary statistic in meta-analyses when studies assess the same outcome but measure the outcome in a variety of ways (e.g., multiple studies measuring depression but using different psychometric scales). Within- and between-study variation or heterogeneity was assessed using Cochran's Q-statistic [32, 33], with a significant $Q$-statistic $(P<0.10)$ indicating heterogeneity among studies. Heterogeneity was also assessed using the $I^{2}$ metric, with higher values denoting a greater degree of heterogeneity (0-40\%: little heterogeneity; 30-60\%: moderate heterogeneity; 50-90\%: substantial heterogeneity; 75-100\%: considerable heterogeneity). $I^{2}$ values $\leq 50 \%$ indicate acceptable heterogeneity among studies [34]. For studies comparing different doses and/or times of drug administration with a single control group, we compared control group data with pooled data from all experimental groups.

Stratified meta-analysis was used to explore the influence of the type of statin, dose, study quality, animal species, type of TBI model, anesthetic agent, and route of drug delivery on estimated effect size [35].

Differences in mean effect sizes were assessed partitioning heterogeneity using the $\chi^{2}$ distribution with $n-1$ degrees of freedom (df). Bonferroni correction was used to adjust significance levels for multiple comparisons (declared significance $=1-(1-$ denoted significance $) \wedge(1 /$ number of comparisons $))$, yielding critical $P$ values of 0.0047 for acquisition memory and 0.0043 for retention memory $[36,37]$.

Metaregression analyses were conducted to reveal potential sources of heterogeneity, as described in a previous study [38]. Covariates included the type of statin, dose, quality of the study, animal species, type of TBI model, anesthetic agent, and route of drug delivery. Due to limited power of our metaregression analyses, we incorporated each covariate separately into the regression model.

The presence of small effect sizes was investigated using funnel plots and Egger's tests. For Egger's tests, a $P$ value of $<0.10$ was considered to indicate the presence of small effect sizes [32].

All statistical analyses were performed using Review Manager (version 5.2) and Stata software (version 12.0). 


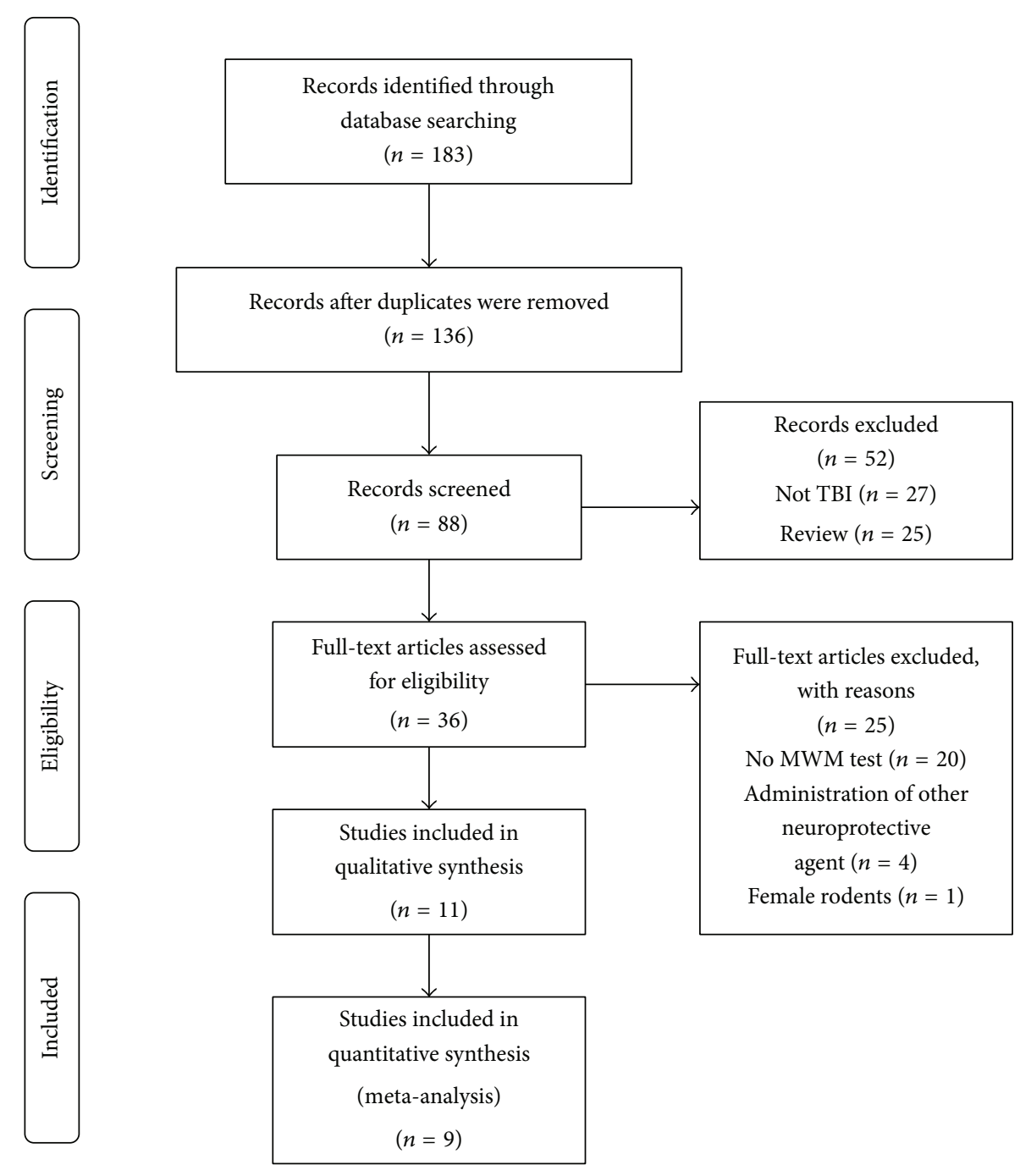

FIGURE 1: Flow diagram of study search process.

\section{Results}

3.1. Study Inclusion. A total of 183 publications were identified, of which 11 met our inclusion criteria $[10,13,16,24-$ 31 . Of these, two were excluded from analysis because they did not report sample size $[24,28]$. Thus, our meta-analysis is based on nine publications, which include 11 comparisons of acquisition memory and 12 comparisons of retention memory (Figure 1).

3.2. Characteristics of Study. Of the 11 included studies (Table 4), three were published in Chinese academic journals [29-31]. Controlled cortical impact injury $[10,13,16,24-$ $26]$ and fluid percussion injury [27, 29-31] were the most frequently used animal models of TBI. Seven studies used rats, three studies used nontransgenic mice, and one study [24] used transgenic mice. Atorvastatin, simvastatin, rosuvastatin, lovastatin, and pravastatin were administered as experimental treatments in doses of $1,2,3$, or $20 \mathrm{mg} / \mathrm{kg} / \mathrm{day}$ via oral gavage or subcutaneous injection. All studies used the MWM to assess cognitive function after TBI.

3.3. Methodological Quality of Studies. Overall, the median quality score for the 11 included studies was poor (5; interquartile range: $4-7$ ), with scores ranging from 3 to 8 . No studies received a score of 0 , and four studies $[10,13$, $25,26]$ received scores indicating high quality (7-10 points). One study [25] did not report randomization of animals into treatment groups. Six studies did not report monitoring of physiological parameters during surgical procedures (although the majority of remaining studies only monitored body or rectal temperature). Only one study [25] assessed dose-response relationships and contained a statement of potential conflict of interests. Four studies [39-42] failed to state that outcome measures were made by experimenters who were blind to animal treatment. Moreover, no study described calculation of necessary sample size. 


\begin{tabular}{|c|c|c|c|c|c|c|c|c|c|c|c|}
\hline 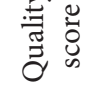 & in & $m$ & $\infty$ & $\wedge$ & $n$ & in & $F$ & $n$ & $r$ & in & in \\
\hline 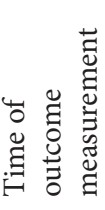 & 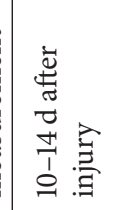 & 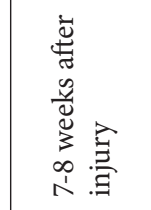 & 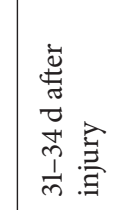 & 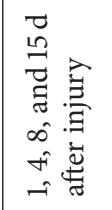 & 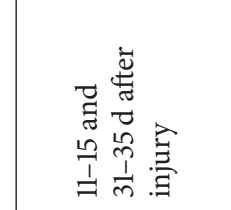 & 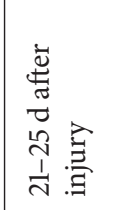 & 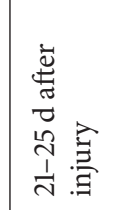 & 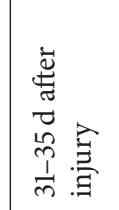 & 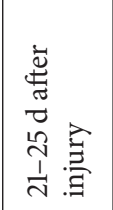 & 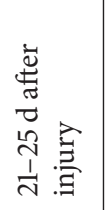 & 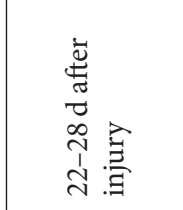 \\
\hline 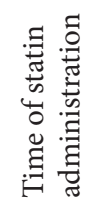 & 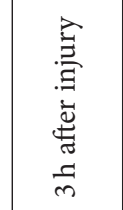 & 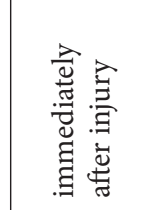 & 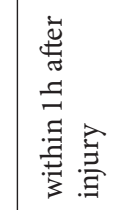 & 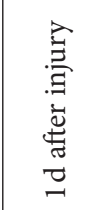 & 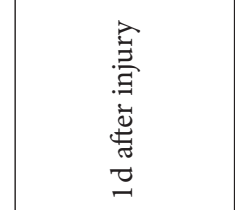 & 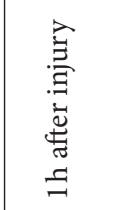 & 苛 & 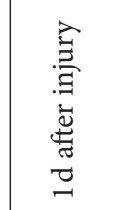 & 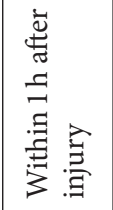 & 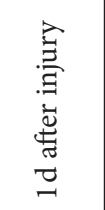 & 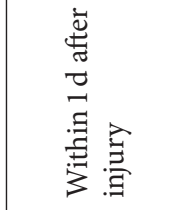 \\
\hline 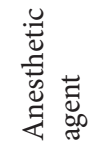 & 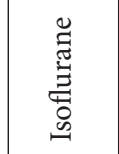 & 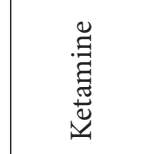 & 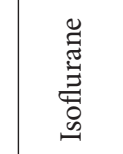 & 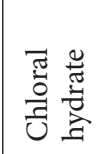 & 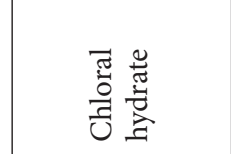 & 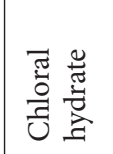 & 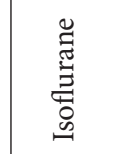 & 吾苛 & 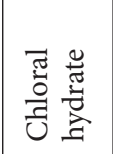 & 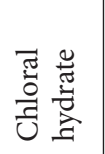 & 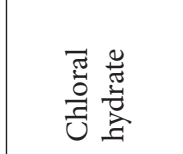 \\
\hline 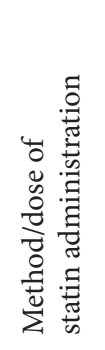 & 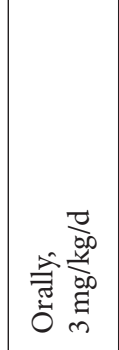 & 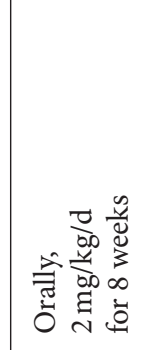 & 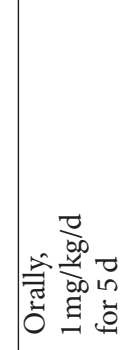 & 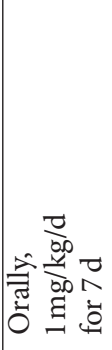 & 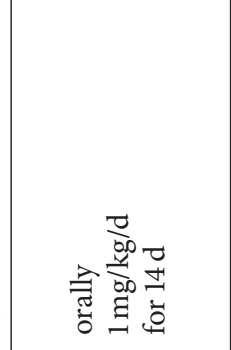 & 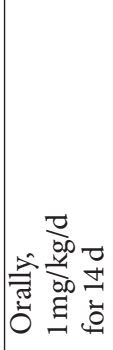 & 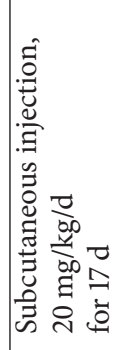 & 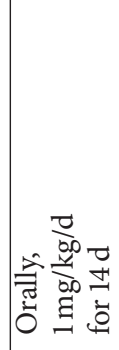 & 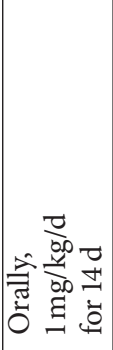 & 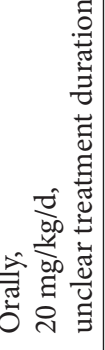 & 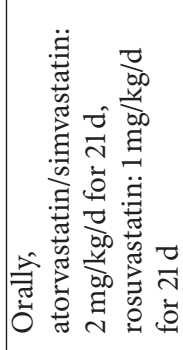 \\
\hline 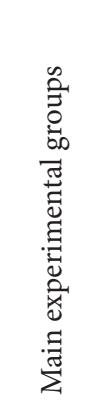 & 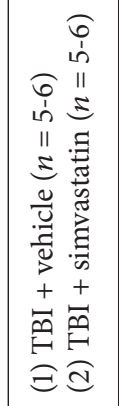 & 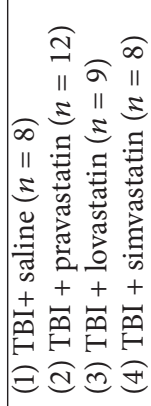 & 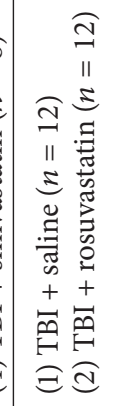 & 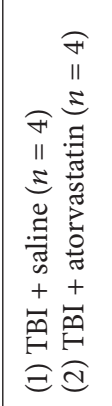 & 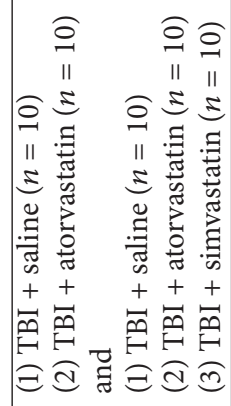 & 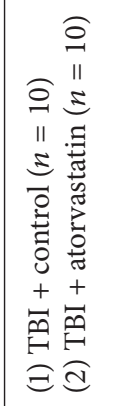 & 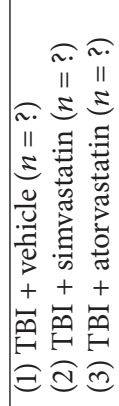 & 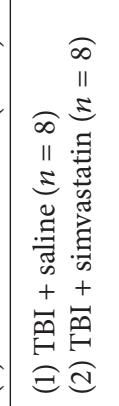 & 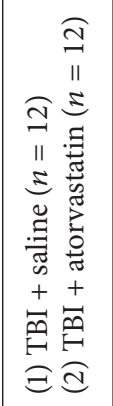 & 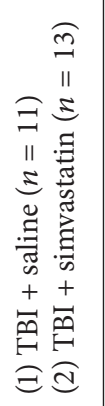 & 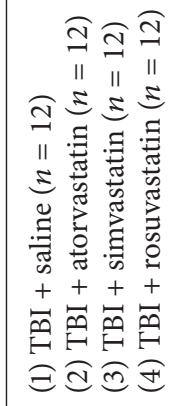 \\
\hline 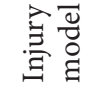 & $\vec{U}$ & $\vec{U}$ & $\vec{U}$ & $\vec{U}$ & $\vec{U}$ & 空 & 急 & $\vec{U}$ & $\overrightarrow{D^{\prime}}$ & $\overrightarrow{\vec{I}^{\prime}}$ & $\overline{\vec{I}}$ \\
\hline 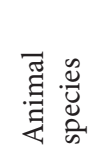 & 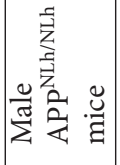 & 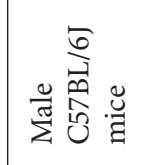 & 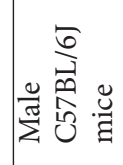 & 营 & 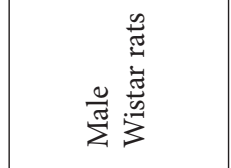 & 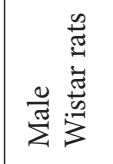 & 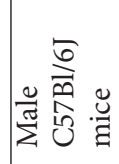 & 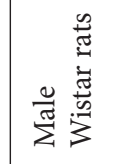 & 苞 & 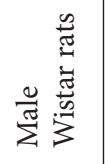 & 苞 \\
\hline & 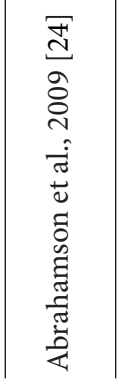 & 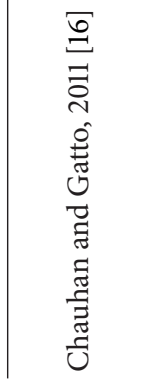 & 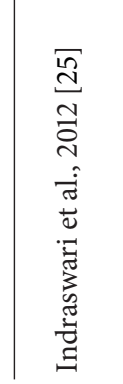 & 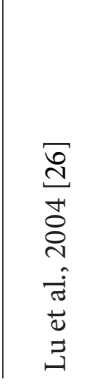 & 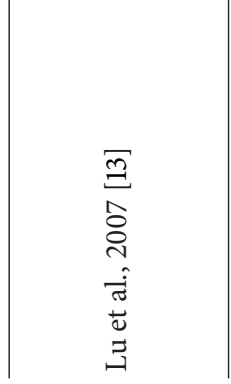 & 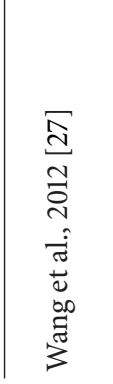 & 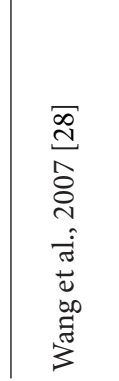 & 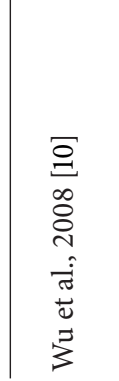 & 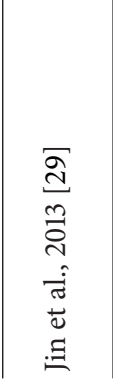 & 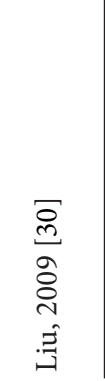 & 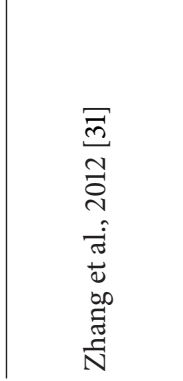 \\
\hline
\end{tabular}




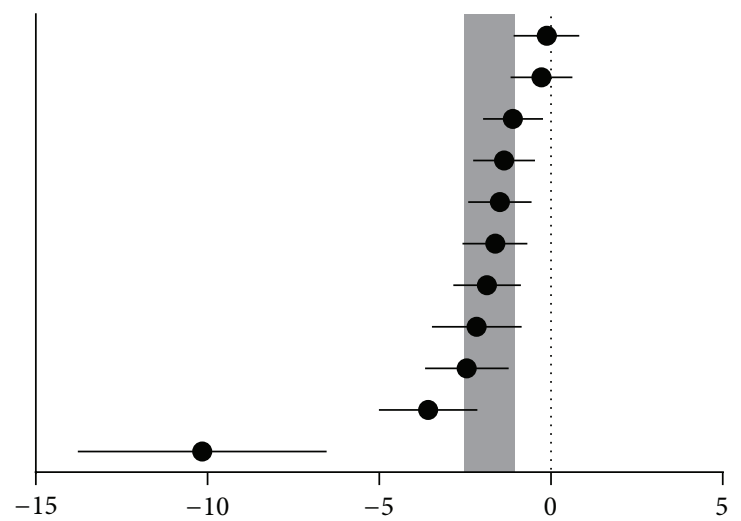

(a)

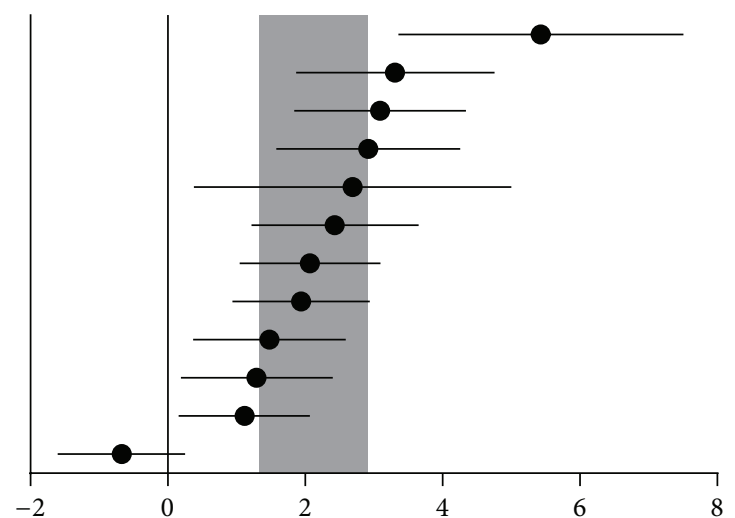

(b)

FIGURE 2: Effects of statins on acquisition memory (a) and retention memory (b). Horizontal lines represent the mean estimated effect size and 95\% CI for each comparison. Vertical gray bars represent the 95\% CI of the pooled estimated effect size.

3.4. Overall Efficacy. For acquisition memory, the global estimated effect of statins was -1.81 (95\% CI: -2.54 to $1.07, P<$ $0.0001)$, with significant heterogeneity among studies $\left(\chi^{2}=\right.$ 49.81, df $=10, P<0.0001, I^{2}=80 \%$; Figure 2(a)). For retention memory, the global estimated effect of statins was 2.12 (95\% CI: 1.33 to $2.9, P<0.0001$ ), with significant heterogeneity among studies $\left(\chi^{2}=55.33, \mathrm{df}=11, P<0.0001\right.$, $I^{2}=80 \%$; Figure 2(b)).

3.5. Stratified Meta-Analysis. In a stratified analysis, trials are grouped according to a particular feature or characteristic and separate meta-analyses are conducted for the trials within each subgroup. The overall summaries of each subgroup can then be inspected for evidence of variation in the effects of the intervention, which would suggest that the stratifying characteristic is an important source of heterogeneity and may moderate treatment efficacy [43].

To compare the efficacy of different types of statins, we examined the protective effects of simvastatin, atorvastatin, and rosuvastatin administration on acquisition memory. We did not include pravastatin and lovastatin in this analysis because of limited data. For retention memory, the effects of simvastatin and atorvastatin administration were examined, and rosuvastatin, pravastatin, and lovastatin were excluded because of limited data. Atorvastatin treatment had a greater beneficial effect on acquisition memory $(-4.55,95 \% \mathrm{CI}:-7.33$ to -1.36$)$ compared with simvastatin $(-1.85,95 \% \mathrm{CI}:-2.38$ to -1.31$)$ or rosuvastatin $(-1.28,95 \% \mathrm{CI}:-1.29$ to -0.65$)$ treatment. For retention memory, simvastatin administration $(2.87,95 \%$ CI: 1.46 to 4.28$)$ had a greater beneficial effect than rosuvastatin administration, although simvastatin effects showed significant heterogeneity among studies $\left(I^{2}=\right.$ $77 \%, P<0.01)$. No significant differences among types of statins were observed ( $P=0.08$ for acquisition memory and $P=0.48$ for retention memory, resp.).

Next, we sought to analyze the efficacy of different doses of statins on cognitive performance. For both acquisition and retention memory, significant beneficial effects were noted for all doses of statins, with a maximum effect at the lowest dose $(-3.93,95 \%$ CI: -6.74 to -1.12 , Figure 3(b); $2.63,95 \%$ CI: 1.75 to 3.52, Figure 4(b), resp.). However, no significant differences among doses were detected $\left(\chi^{2}=2.93, \mathrm{df}=1\right.$, and $P=0.09 ; \quad \chi^{2}=2.37, \mathrm{df}=1$, and $P=0.12$, resp.).

The effect sizes for acquisition and retention memory were also examined relative to study quality score. No significant differences in effect sizes were observed between lowerscored and higher-scored studies $\left(\chi^{2}=10.63, \mathrm{df}=4\right.$, and $P=$ $0.03 ; \chi^{2}=5.68, \mathrm{df}=2$, and $P=0.06$ for acquisition and retention memory, resp.). However, effect size for acquisition memory was maximum for studies with a quality score of 4 (-2.94, 95\% CI: -4.04 to -1.85 ; Figure 3(c)), and effect size for retention memory was higher for studies with a quality score of 7 (2.84, 95\% CI: 1.49 to 4.19; Figure 4(c)) than those with scores of 3 or 5 .

For acquisition memory, effect size was similar for experiments using male Wistar rats and those using male C57BL/6J mice $\left(\chi^{2}=1.36, \mathrm{df}=1\right.$, and $P=0.24$; Figure $\left.3(\mathrm{~d})\right)$. However, for retention memory, effect size was higher for studies using male Wistar rats $\left(2.58,95 \% \mathrm{CI}: 1.90\right.$ to $3.52 ; \chi^{2}=5.6, \mathrm{df}=1$, and $P=0.02$; Figure $4(\mathrm{~d}))$.

Concerning anesthetic agents and types of TBI models, for acquisition memory, effect size was significantly higher in studies using closed head injury models $(-3.57,95 \% \mathrm{CI}$ : -5.01 to $-2.14 ; \chi^{2}=12.09, \mathrm{df}=2$, and $P=0.002$; Figure 5 (a)) and those using isoflurane anaesthesia $(-2.27,95 \% \mathrm{CI}:-4.68$ to $-0.15 ; \quad \chi^{2}=3.93, \mathrm{df}=2$, and $P=0.14$; Figure $\left.5(\mathrm{~b})\right)$. For retention memory, effect size was significantly higher in studies using fluid percussion injury models $(2.39,95 \% \mathrm{CI}$ : 1.82 to $2.90 ; \chi^{2}=0.42, \mathrm{df}=1$, and $P=0.52$; Figure $\left.6(\mathrm{a})\right)$ and those using chloral hydrate anaesthesia $(2.58,95 \% \mathrm{CI}: 1.90$ to 3.25; $\chi^{2}=5.60, \mathrm{df}=1$, and $P=0.02$; Figure $6(\mathrm{~b})$ ).

For acquisition memory, intraperitoneal administration (-3.57, 95\% CI: -5.01 to -2.14 ; Figure 5(c)) was associated with a greater beneficial outcome than oral administration, but there were no significant differences between routes of administration $\left(\chi^{2}=5.69, \mathrm{df}=1\right.$, and $\left.P=0.02\right)$. 


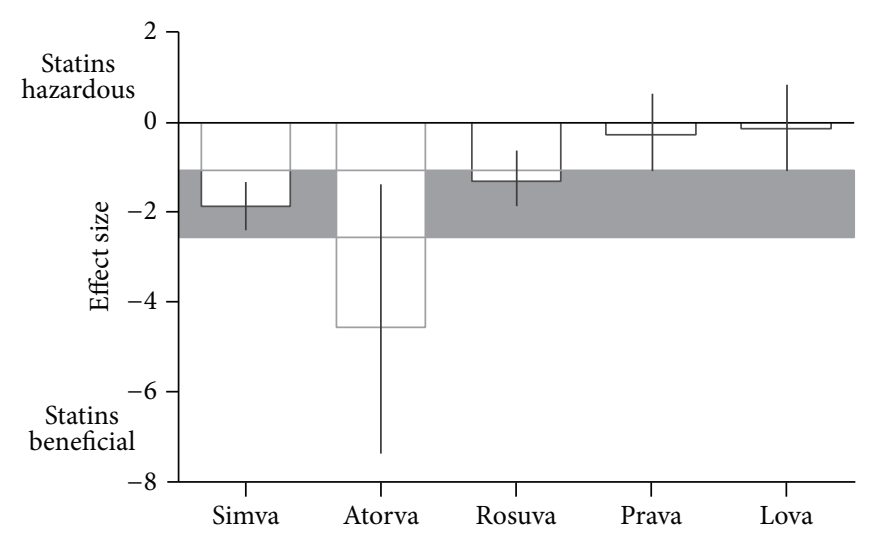

(a)

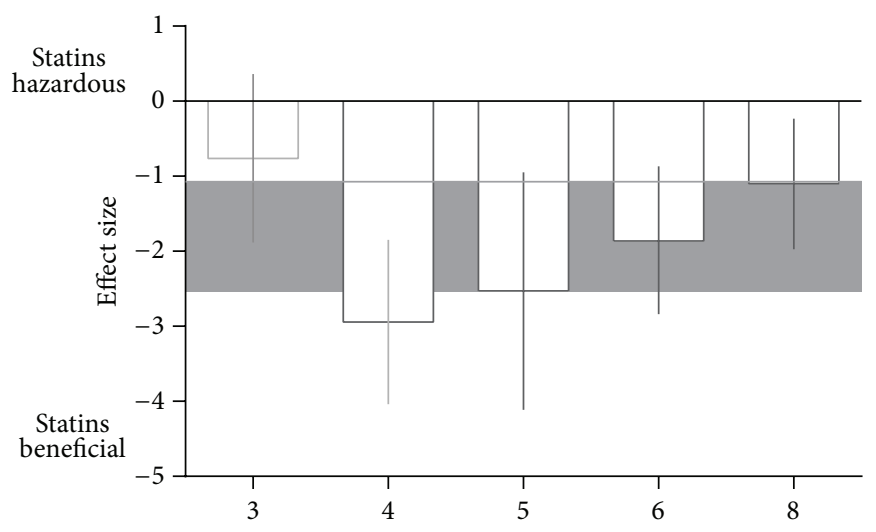

(c)

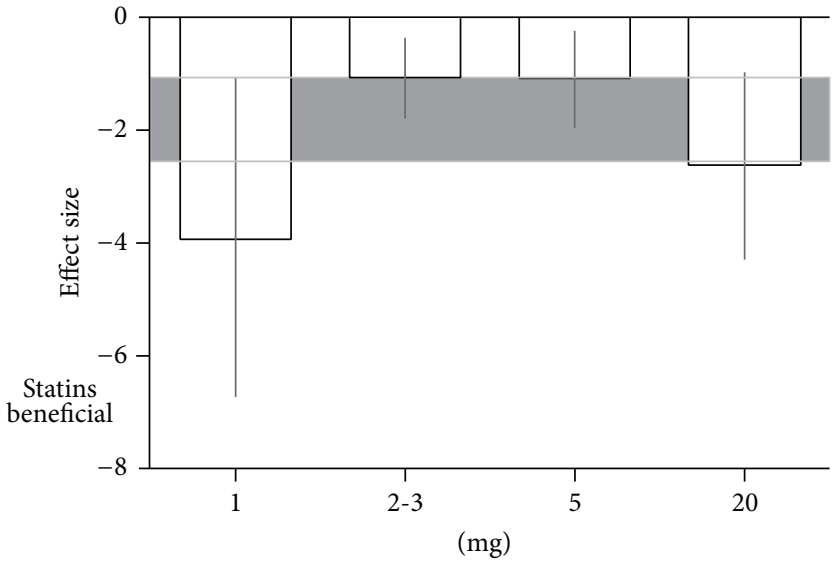

(b)

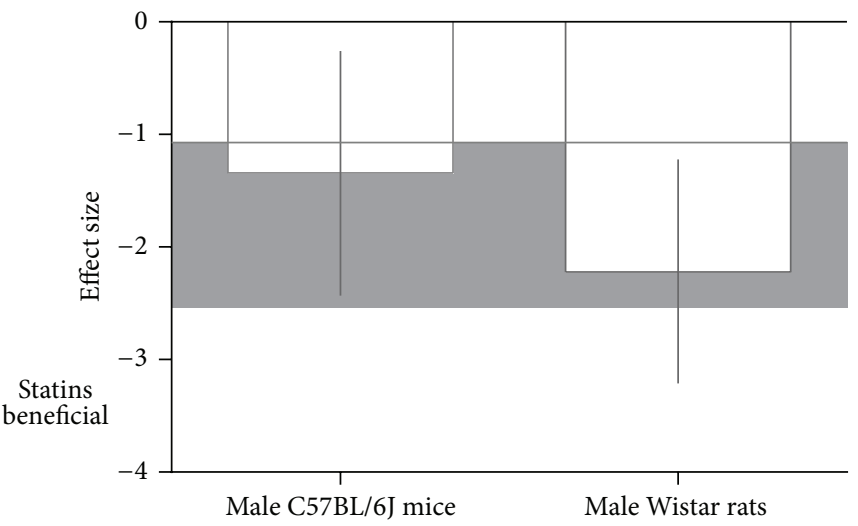

(d)

FIGURE 3: Effect size for acquisition memory stratified by (a) type of statin, (b) dose, (c) quality of study, and (d) animal species. Grey bands represent the $95 \%$ CI for the global estimated effect size.

3.6. Metaregression Analyses. Metaregression is an extension of subgroup analysis that allows investigation of the effect of continuous as well as categorical characteristics. In principle, metaregression also allows investigation of the effects of multiple factors simultaneously. The outcome variable is the effect estimate, and the explanatory variables are characteristics of studies that might influence effect size, which are often called "potential effect modifiers" or covariates.

To further explore heterogeneity among studies, metaregression was conducted for acquisition and retention memory. For retention memory, animal species, quality score, and type of anesthetic agent were significant sources of heterogeneity $(P<0.05)$. However, for acquisition memory, heterogeneity was independent of these factors (Table 5).

3.7. Publication Bias. Finally, we sought to identify the presence of small study effects, which may contribute to publication bias. Funnel plots show asymmetry for both acquisition and retention memory data, indicating evidence of small study effects (Figures 7(a) and 7(b); Egger regression, $P<$ 0.0001 and $P=0.007$, resp.).
3.8. Possible Drug Protection Mechanism Analysis. All studies selected during initial screening assessed the biological mechanisms of statin activity. Across studies, the neuroprotective effect of statins was attributed primarily to regulation of circulating endothelial progenitor cells and angiogenesis, increased neurogenesis and reduced neuronal degeneration, intravascular thrombosis and inflammation, and reduced microglial activation (Table 6).

\section{Discussions}

The results of animal experiments are used to inform decisions regarding the design and conduct of subsequent clinical trials. Systematic reviews of animal studies can allow such decisions to be based on the entirety of existing evidence that is synthesized in an unbiased manner. We therefore systematically reviewed and collated experimental evidence of the effect of statin administration before or after TBI in animal models, determined the efficacy of statin treatment in TBI, and explored the impact of study characteristics on statin efficacy. 


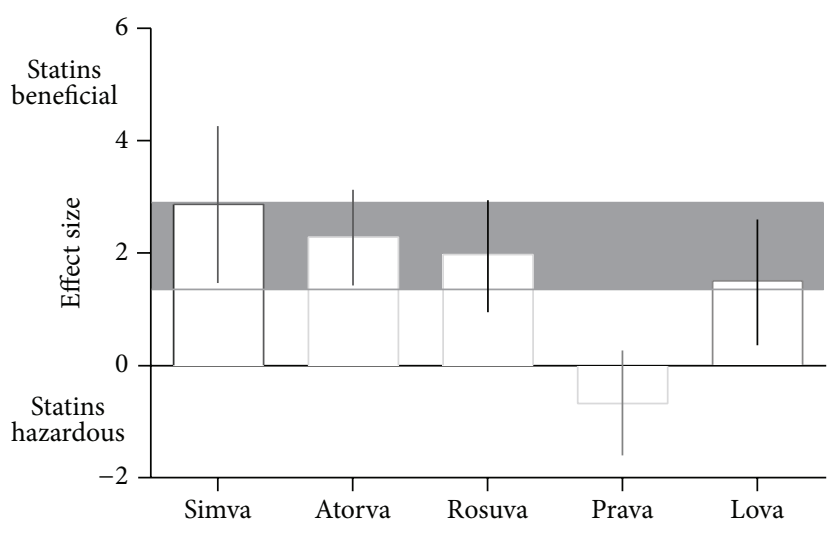

(a)

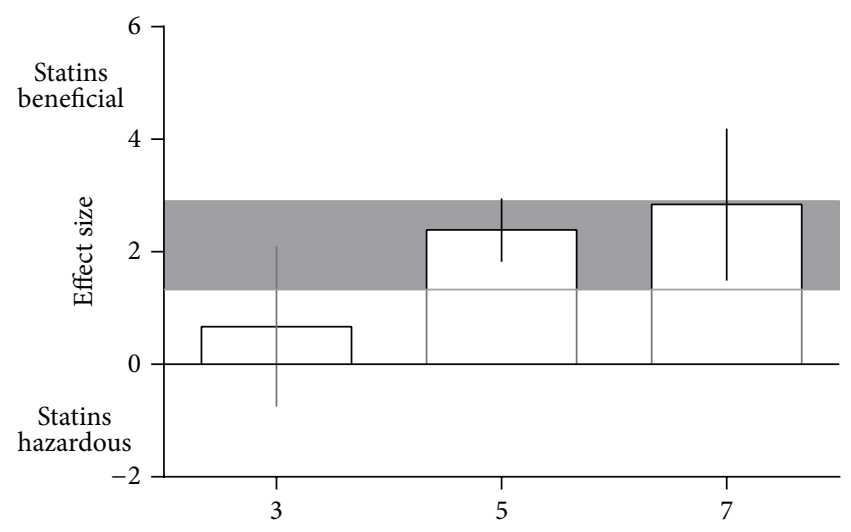

(c)

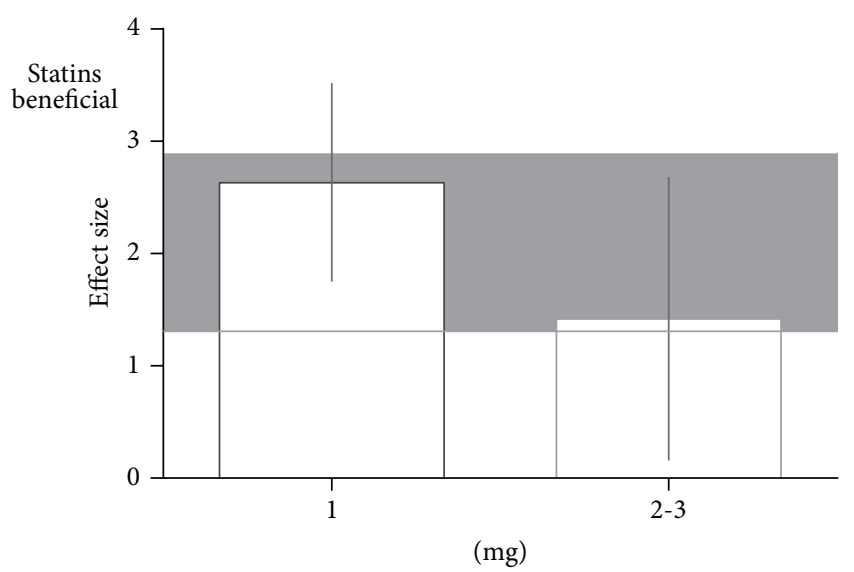

(b)

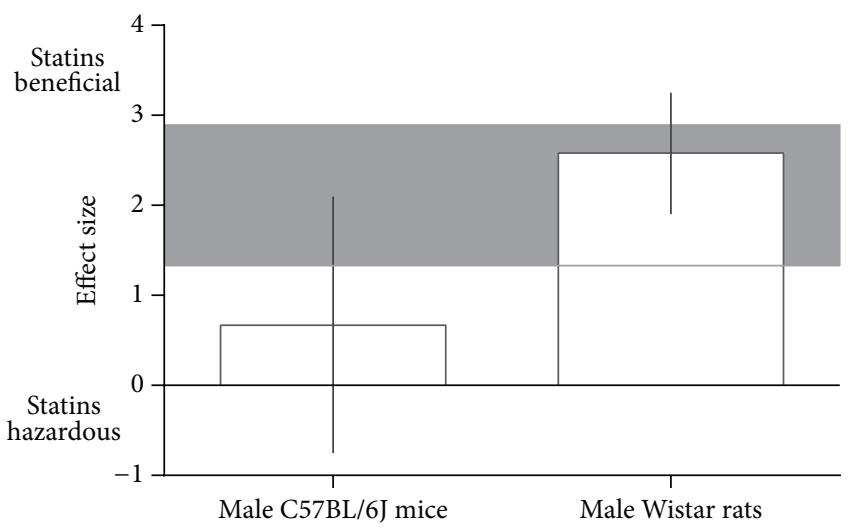

(d)

FIGURE 4: Effect size for retention memory stratified by (a) type of statin, (b) dose, (c) quality of study, and (d) animal species. Grey bands represent the $95 \%$ CI for the global estimated effect size.

Although there are some systematic reviews of pharmacological treatments (i.e., beta- 2 receptor antagonists, progesterone) for TBI in animal models $[44,45]$, to our knowledge, this investigation is the first systematic review and metaanalysis of the efficacy of statins on cognitive deficits in animal models of TBI. Despite the presence of small effects and statistical heterogeneity among studies, our investigation shows that statins potentially exert neuroprotective effects in terms of improving cognitive outcome after TBI, with atorvastatin exerting the most protective effect on acquisition memory and simvastatin exerting the most protective effect on retention memory. Moreover, statin treatment provides better neuroprotection of acquisition memory for closed head injury. However, stratified analysis detected no significant influence of study quality, statin dose, animal species, drug delivery route, or anesthetic agent. Similar works [23, 46] have been performed in the context of experimental stroke, which demonstrate the neuroprotective effects of statins on animal stroke models in terms of reduced infarct volume and improved neurological severity score. Although stroke and TBI are different conditions, many aspects of their pathologies are similar, and these investigations provide further evidence of the neuroprotective effects of statins, thereby supporting their potential use for human TBI therapy.

We assessed the methodological quality of studies in accordance with previously described standards for preclinical development of neuroprotective drugs with minor modifications [22]. Overall, we found that the quality of the included studies was poor, as many failed to report blinded assessment of outcome or to determine a dose-response relationship, which are important issues that are generally required in clinical studies [47]. Moreover, lower quality studies showed a trend toward better acquisition memory outcomes. Therefore, the global estimated effect of statins on cognition may be overstated in low quality studies.

Furthermore, we found significant heterogeneity among study results. The main reasons for heterogeneity were the limited number of studies and the small sample sizes within those studies. Another important contribution to this heterogeneity may be the low quality of studies and potential bias 


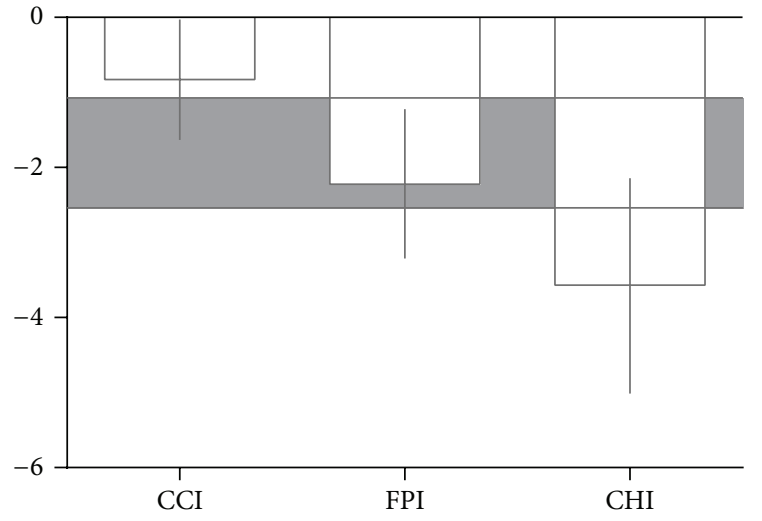

(a)

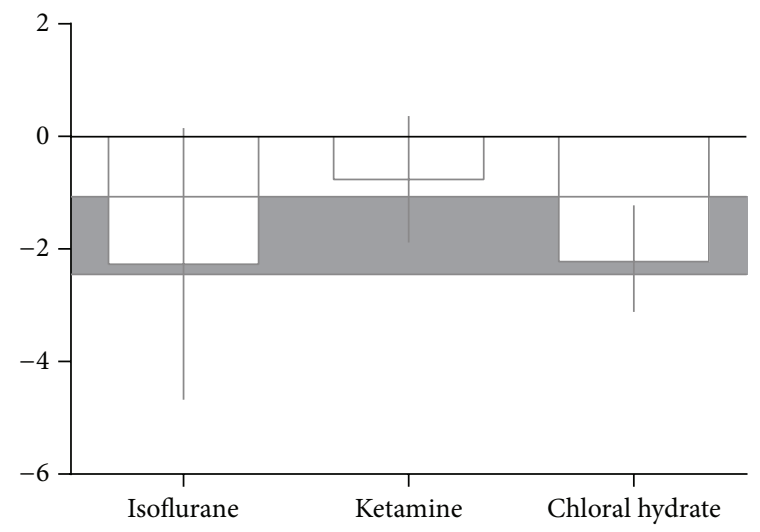

(b)

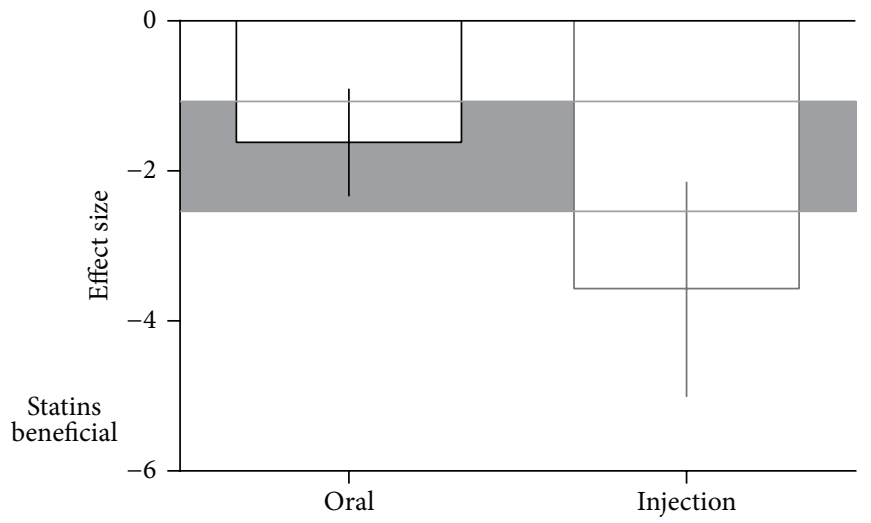

(c)

FIGURE 5: Effect size for acquisition memory stratified by (a) method of TBI induction, (b) anesthetic agent, and (c) route of drug delivery. Grey bands represent the $95 \%$ CI for the global estimated effect size.

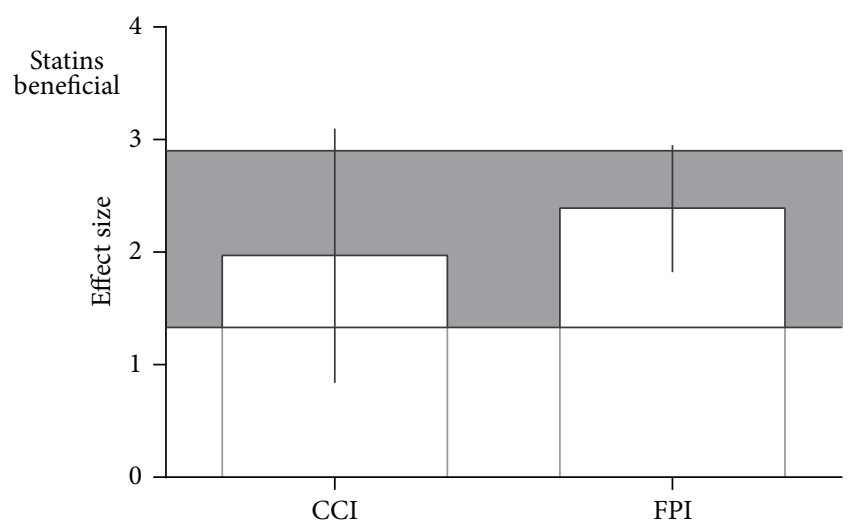

(a)

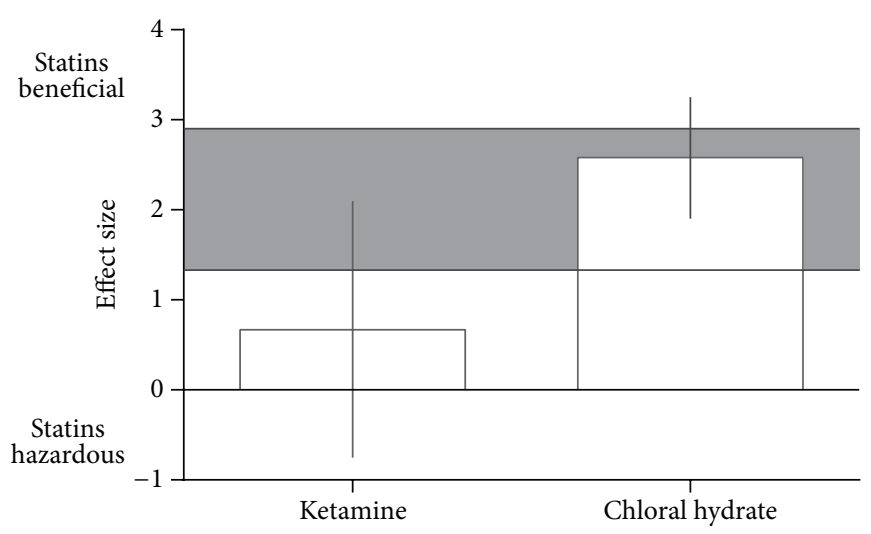

(b)

FIGURE 6: Effect size for retention memory stratified by (a) method of TBI induction and (b) anesthetic agent. Grey bands represent the 95\% CI for the global estimated effect size.

of the studies selected for analysis [48]. To examine potential sources of heterogeneity, we performed metaregression analysis. Unfortunately, for retention memory, the adjusted $R^{2}$ was negative (data not shown) because the number of studies was small and the covariates explained less heterogeneity than would be expected by chance [49]. Therefore, it was not possible to accurately judge whether the heterogeneity we observed was independent of these factors, which made the analysis less reliable.

Our study has several limitations, which are also observed in previous systematic reviews of animal studies [44, 50,51]. First, our analysis is only based on published data and did not 
TABLE 5: Metaregression analysis to identify sources of bias associated with study characteristics.

(a) Acquisition memory

\begin{tabular}{|c|c|c|c|c|c|c|}
\hline \multirow{2}{*}{$\begin{array}{l}\text { Covariates } \\
\text { Species }\end{array}$} & \multirow{2}{*}{$\begin{array}{c}\text { Coef. } \\
-1.218926\end{array}$} & \multirow{2}{*}{$\begin{array}{c}\text { Std. err. } \\
1.425615\end{array}$} & \multirow{2}{*}{$\frac{t}{-0.86}$} & \multirow{2}{*}{$\begin{array}{c}P>|t| \\
0.415\end{array}$} & \multicolumn{2}{|c|}{ [95\% conf. interval] } \\
\hline & & & & & -4.443892 & 2.00604 \\
\hline Quality & -.1059611 & .5134567 & -0.21 & 0.841 & -1.267481 & 1.055559 \\
\hline Route & 1.794537 & 2.46653 & 0.73 & 0.485 & -3.785142 & 7.374215 \\
\hline \multicolumn{7}{|l|}{ Statins } \\
\hline Atorva & -4.098033 & 2.606752 & -1.57 & 0.167 & -10.47653 & 2.28046 \\
\hline Lova & .1509175 & 3.110633 & 0.05 & 0.963 & -7.460527 & 7.762362 \\
\hline Prava & -1.058402 & 2.688579 & -0.39 & 0.707 & -7.637118 & 5.520313 \\
\hline Simva & -1.752053 & 2.462111 & -0.71 & 0.503 & -7.776621 & 4.272516 \\
\hline \multicolumn{7}{|l|}{ Dose } \\
\hline $1 \mathrm{mg}$ & -2.813334 & 2.734279 & -1.03 & 0.338 & -9.278877 & 3.652209 \\
\hline $2 \mathrm{mg}$ & -.0024291 & 2.536972 & -0.00 & 0.999 & -6.001415 & 5.996557 \\
\hline $20 \mathrm{mg}$ & -1.646112 & 2.854527 & -0.58 & 0.582 & -8.395994 & 5.103771 \\
\hline \multicolumn{7}{|l|}{ Anaesthetic used } \\
\hline Chloral hydrate & -.3426693 & 2.006333 & -0.17 & 0.869 & -4.969281 & 4.283942 \\
\hline Ketamine & 1.502998 & 2.222102 & 0.68 & 0.518 & -3.621178 & 6.627174 \\
\hline \multicolumn{7}{|l|}{ Injury model } \\
\hline CCI & 2.776583 & 2.521451 & 1.10 & 0.303 & -3.037893 & 8.591059 \\
\hline FPI & 1.121622 & 2.456371 & 0.46 & 0.660 & -4.54278 & 6.786024 \\
\hline
\end{tabular}

(b) Retention memory

\begin{tabular}{|c|c|c|c|c|c|c|}
\hline Covariates & Coef. & Std. err. & $t$ & $P>|t|$ & \multicolumn{2}{|c|}{ [95\% conf. interval] } \\
\hline Species & -2.045101 & .7652448 & -2.67 & 0.023 & -3.750172 & -.3400288 \\
\hline Dose & -1.358647 & .8065987 & -1.68 & 0.123 & -3.155861 & .4385665 \\
\hline Quality & .5416233 & .2248974 & 2.41 & 0.037 & .0405206 & 1.042726 \\
\hline Anaesthetic used & 2.045101 & .7652448 & 2.67 & 0.023 & .3400288 & 3.750172 \\
\hline Injury model & -.5074523 & .9452993 & -0.54 & 0.603 & -2.61371 & 1.598806 \\
\hline \multicolumn{7}{|l|}{ Statins } \\
\hline Atorva & .4747928 & 1.355065 & 0.35 & 0.736 & -2.729427 & 3.679012 \\
\hline Lova & -.4478023 & 1.743837 & -0.26 & 0.805 & -4.571322 & 3.675718 \\
\hline Prava & -2.709569 & 1.71473 & -1.58 & 0.158 & -6.764262 & 1.345123 \\
\hline Simva & .9555847 & 1.387517 & 0.69 & 0.513 & -2.325371 & 4.23654 \\
\hline
\end{tabular}

TABle 6: Possible protective mechanisms of statins.

\begin{tabular}{|c|c|}
\hline Possible protective mechanisms of statins & Studies \\
\hline Blunted TBI-induced increases in amyloid beta protein, reduced hippocampal tissue damage, and microglial activation & {$[24]$} \\
\hline Restored axonal integrity & {$[16]$} \\
\hline $\begin{array}{l}\text { Downregulation of inflammatory gene expression, reduced neuronal degeneration, preserved neuronal density, and } \\
\text { reduced microgliosis }\end{array}$ & {$[25]$} \\
\hline Reduction of intravascular thrombosis, increased cerebral microvascular patency and integrity & {$[26]$} \\
\hline Increased neurogenesis in the dentate gyrus, reduced delayed neuronal death in the hippocampal CA3 region & {$[10,13]$} \\
\hline Reduced hippocampal degeneration, improved cerebral blood flow & {$[28]$} \\
\hline Regulation of circulating endothelial progenitor cells and angiogenesis & {$[27,29-31]$} \\
\hline
\end{tabular}

take unpublished data into account; therefore publication bias should be considered. Second, we focused only on the effect of statins on cognitive deficits following TBI, largely due to insufficient data regarding histopathology such as lesion volume. Functional outcome, in combination with effects on histopathology, may be as important in terms of assessing benefit of potential neuroprotective drugs [52]. Third, the current findings may be influenced by the selective inclusion of studies that examined only male rodents. Although intact male and female animals should be examined 


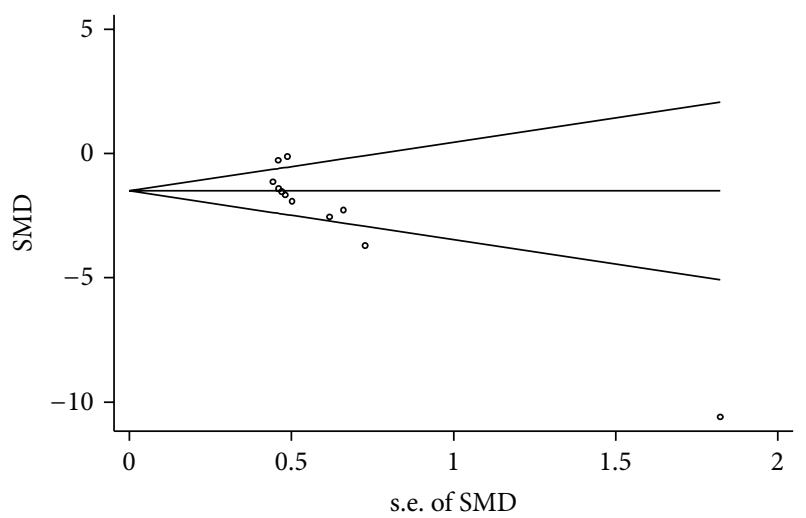

(a) Begg's funnel plot with pseudo 95\% confidence limits

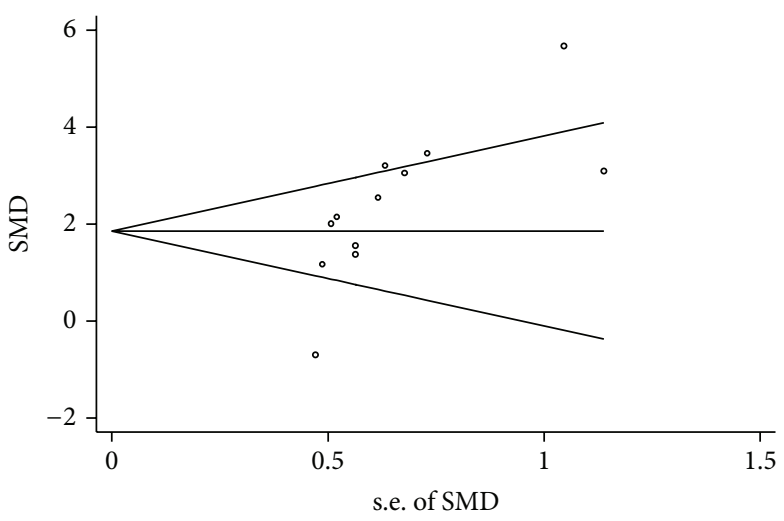

(b) Begg's funnel plot with pseudo $95 \%$ confidence limits

FIGURE 7: Funnel plot for acquisition memory (a) and retention memory (b).

prior to clinical investigation, the small number of studies that examined intact females, combined with potential sex differences in outcome [53], meant that their inclusion could have muddied the results [51]. Fourth, a variety of different metrics were used (e.g., pressure, weight, and velocity) to evaluate TBI severity, and no studies specified the degree of severity (e.g., mild, moderate, or severe). Thus, the results of different studies could be more accurately compared if injury severity is reported in a consistent manner. Fifth, although we found that statin treatment can have beneficial effects in animal models of TBI, the majority of studies used only controlled cortical impact or fluid percussion injury models. However, any one animal model may not fully recapitulate all the aspects of secondary injury development observed in humans with TBI [54], thereby limiting the extent to which this experimental research translates to a clinical population. Finally, there were large numbers of studies that failed to report, or provide upon written request, the necessary data, which therefore had to be derived from graphs. Although we enlarged the graphs, and data were independently extracted by two investigators, this technique can be imprecise. Moreover, extracting multiple pieces of information from a single publication has the potential to introduce bias into systematic reviews because the results are generated by the same investigators.

To improve the transition from animal experiments to human clinical trials, future animal studies of statins or other drugs should improve their methodological reporting and quality control as follows: (1) additional appropriate and standardized TBI models are needed to evaluate the impact of promising pharmacological interventions; (2) treatment efficacy should be tested in both sexes and different species (i.e., rabbits, cats, or gyrencephalic primates); (3) researchers should consult and follow the ARRIVE guidelines [55, 56] when designing studies and report full methodological details to allow others to reproduce and validate their results and to enable more accurate reviews and meta-analyses; (4) other short- and long-term outcomes such as lesion volume, brain edema, blood-brain barrier permeability, and depression-like behavior should also be examined.

\section{Conclusions}

Despite its limitations, this systematic review and metaanalysis demonstrates that statins could reduce cognitive deficits in animal models of TBI. A fundamental assumption is that the results of animal studies, if performed well enough, will predict effects in humans. However, promising neuroprotective drugs previously identified as effective in animal TBI models have failed in Phase II or III clinical trials [54]. Therefore, without rigorous, robust, and detailed preclinical evaluation, it is unlikely that novel neuroprotective drugs will prove effective when tested in large, time-consuming, and expensive human clinical trials, thereby warranting further well-designed and well-reported experimental animal studies.

\section{Conflict of Interests}

The authors declare that there is no conflict of interests regarding the publication of this paper.

\section{Acknowledgments}

This work was financially supported by the key-discipline construct programs of Hunan province and SATCM. The authors are grateful to Simiao Wu and Ruili Wei for their kind help.

\section{References}

[1] V. L. Feigin, A. Theadom, S. Barker-Collo et al., "Incidence of traumatic brain injury in New Zealand: a population-based study," The Lancet Neurology, vol. 12, no. 1, pp. 53-64, 2013.

[2] K. R. Walker and G. Tesco, "Molecular mechanisms of cognitive dysfunction following traumatic brain injury," Frontiers in Aging Neuroscience, vol. 5, article 29, 2013.

[3] S. S. Dikmen, J. D. Corrigan, H. S. Levin, J. MacHamer, W. Stiers, and M. G. Weisskopf, "Cognitive outcome following traumatic brain injury," Journal of Head Trauma Rehabilitation, vol. 24, no. 6, pp. 430-438, 2009. 
[4] A. R. Rabinowitz and H. S. Levin, "Cognitive sequelae of traumatic brain injury," Psychiatric Clinics of North America, vol. 37, no. 1, pp. 1-11, 2014.

[5] D. K. Menon, "Exploring new routes for neuroprotective drug development in traumatic brain injury," Science Translational Medicine, vol. 2, no. 27, p. 27rv21, 2010.

[6] J. Lu, J. M. Frerich, L. C. Turtzo et al., "Histone deacetylase inhibitors are neuroprotective and preserve NGF-mediated cell survival following traumatic brain injury," Proceedings of the National Academy of Sciences of the United States of America, vol. 110, no. 26, pp. 10747-10752, 2013.

[7] K. Stepień, M. Tomaszewski, and S. J. Czuczwar, "Neuroprotective properties of statins," Pharmacological Reports, vol. 57, no. 5, pp. 561-569, 2005.

[8] J. K. Liao and U. Laufs, "Pleiotropic effects of statins," Annual Review of Pharmacology and Toxicology, vol. 45, pp. 89-118, 2005.

[9] H. Wu, D. Lu, H. Jiang et al., "Increase in phosphorylation of Akt and its downstream signaling targets and suppression of apoptosis by simvastatin after traumatic brain injury: laboratory investigation," Journal of Neurosurgery, vol. 109, no. 4, pp. 691698, 2008.

[10] H. Wu, D. Lu, H. Jiang et al., "Simvastatin-mediated upregulation of VEGF and BDNF, activation of the PI3K/Akt pathway, and increase of neurogenesis are associated with therapeutic improvement after traumatic brain injury," Journal of Neurotrauma, vol. 25, no. 2, pp. 130-139, 2008.

[11] G. Chen, S. Zhang, J. Shi, J. Ai, M. Qi, and C. Hang, "Simvastatin reduces secondary brain injury caused by cortical contusion in rats: possible involvement of TLR4/NF- $\kappa$ B pathway," Experimental Neurology, vol. 216, no. 2, pp. 398-406, 2009.

[12] B. Li, A. Mahmood, D. Lu et al., "Simvastatin attenuates microglial cells and astrocyte activation and decreases interleukin- $1 \beta$ level after traumatic brain injury," Neurosurgery, vol. 65, no. 1 , pp. 179-185, 2009.

[13] D. Lu, C. Qu, A. Goussev et al., "Statins increase neurogenesis in the dentate gyrus, reduce delayed neuronal death in the hippocampal CA3 region, and improve spatial learning in rat after traumatic brain injury," Journal of Neurotrauma, vol. 24, no. 7, pp. 1132-1146, 2007.

[14] O. F. Turkoglu, H. Eroglu, O. Okutan et al., "Atorvastatin efficiency after traumatic brain injury in rats," Surgical Neurology, vol. 72, no. 2, pp. 146-152, 2009.

[15] S. Chen, T. Hung, C. Chen et al., "Lovastatin improves histological and functional outcomes and reduces inflammation after experimental traumatic brain injury," Life Sciences, vol. 81, no. 4, pp. 288-298, 2007.

[16] N. B. Chauhan and R. Gatto, "Restoration of cognitive deficits after statin feeding in TBI," Restorative Neurology and Neuroscience, vol. 29, no. 1, pp. 23-34, 2011.

[17] K. Richardson, M. Schoen, B. French et al., "Statins and cognitive function: a systematic review," Annals of Internal Medicine, vol. 159, no. 10, pp. 688-697, 2013.

[18] E. Bruckert, G. Hayem, S. Dejager, C. Yau, and B. Bégaud, "Mild to moderate muscular symptoms with high-dosage statin therapy in hyperlipidemic patients - the PRIMO study," Cardiovascular Drugs and Therapy, vol. 19, no. 6, pp. 403-414, 2005.

[19] M. L. Ancelin, I. Carrìere, P. Barberger-Gateau et al., "Lipid lowering agents, cognitive decline, and dementia: the three-city study," Journal of Alzheimer's Disease, vol. 30, no. 3, pp. 629-637, 2012.

[20] J. Benito-León, E. D. Louis, S. Vega, and F. Bermejo-Pareja, "Statins and cognitive functioning in the elderly: a populationbased study," Journal of Alzheimer's Disease, vol. 21, no. 1, pp. 95-102, 2010.

[21] S. Trompet, P. Van Vliet, A. J. M. De Craen et al., "Pravastatin and cognitive function in the elderly. Results of the PROSPER study," Journal of Neurology, vol. 257, no. 1, pp. 85-90, 2010.

[22] M. R. Macleod, T. O'Collins, D. W. Howells, and G. A. Donnan, "Pooling of animal experimental data reveals influence of study design and publication bias," Stroke, vol. 35, no. 5, pp. 1203-1208, 2004.

[23] L. García-Bonilla, M. Campos, D. Giralt et al., "Evidence for the efficacy of statins in animal stroke models: a meta-analysis," Journal of Neurochemistry, vol. 122, no. 2, pp. 233-243, 2012.

[24] E. E. Abrahamson, M. D. Ikonomovic, C. Edward Dixon, and S. T. DeKosky, "Simvastatin therapy prevents brain traumainduced increases in $\beta$-amyloid peptide levels," Annals of Neurology, vol. 66, no. 3, pp. 407-414, 2009.

[25] F. Indraswari, H. Wang, B. Lei et al., "Statins improve outcome in murine models of intracranial hemorrhage and traumatic brain injury: a translational approach," Journal of Neurotrauma, vol. 29, no. 7, pp. 1388-1400, 2012.

[26] D. Lu, A. Mahmood, A. Goussev et al., "Atorvastatin reduction of intravascular thrombosis, increase in cerebral microvascular patency and integrity, and enhancement of spatial learning in rats subjected to traumatic brain injury," Journal of Neurosurgery, vol. 101, no. 5, pp. 813-821, 2004.

[27] B. Wang, L. Sun, Y. Tian et al., "Effects of atorvastatin in the regulation of circulating EPCs and angiogenesis in traumatic brain injury in rats," Journal of the Neurological Sciences, vol. 319, no. 1-2, pp. 117-123, 2012.

[28] H. Wang, J. R. Lynch, P. Song et al., "Simvastatin and atorvastatin improve behavioral outcome, reduce hippocampal degeneration, and improve cerebral blood flow after experimental traumatic brain injury," Experimental Neurology, vol. 206, no. 1, pp. 59-69, 2007.

[29] W. Jin, B. Wang, Z. Zhao et al., "Atorvastatin regulates endothelial progenitor cells and improves spatial cognition and memory function in rats with traumatic brain injury," Guangdong Medical Journal, vol. 34, no. 6, pp. 835-837, 2013.

[30] Q. Liu, The Effects of Simvastatin on the Mobilization of Endothelial Progenitor Cells and Angiogenesis of Brain Tissue of Rats after Traumatic Brain Injury: An Experimental Study, Tianjin Medical University, 2009.

[31] Y. Zhang, L. Liu, Z. Zhao, and J. Zhang, "Effects of statins in the regulation of circulating EPCs and cognition and memory function in rats with traumatic brain injury," Shandong Medical Journal, vol. 52, no. 36, pp. 51-54, 105, 2012.

[32] M. Egger, G. D. Smith, M. Schneider, and C. Minder, "Bias in meta-analysis detected by a simple, graphical test," The British Medical Journal, vol. 315, no. 7109, pp. 629-634, 1997.

[33] H. S. Sacks, J. Berrier, D. Reitman, V. A. Ancona-Berk, and T. C. Chalmers, "Meta-analyses of randomized controlled trials," The New England Journal of Medicine, vol. 316, no. 8, pp. 450-455, 1987.

[34] J. Higgins and S. Green, Cochrane Handbook for Systematic Reviews of Interventions, Version 5.1.0, The Cochrane Collaboration, 2011. 
[35] H. M. Vesterinen, E. S. Sena, K. J. Egan et al., "Meta-analysis of data from animal studies: a practical guide," Journal of Neuroscience Method, vol. 221, pp. 92-102, 2014.

[36] H. M. Vesterinen, G. L. Currie, S. Carter et al., "Systematic review and stratified meta-analysis of the efficacy of RhoA and Rho kinase inhibitors in animal models of ischaemic stroke," Systematic Reviews, vol. 2, p. 33, 2013.

[37] M. Borenstein, Introduction to Meta-Analysis, John Wiley \& Sons, Chichester, UK, 2009.

[38] J. B. Liu, M. Li, H. Chen et al., "Association of vitiligo with HLA-A2: a meta-analysis," Journal of the European Academy of Dermatology and Venereology, vol. 21, no. 2, pp. 205-213, 2007.

[39] G. Grasso, A. Sfacteria, F. Meli, V. Fodale, M. Buemi, and D. G. Iacopino, "Neuroprotection by erythropoietin administration after experimental traumatic brain injury," Brain Research, vol. 1182, no. 1, pp. 99-105, 2007.

[40] Y. Xiong, A. Mahmood, D. Lu et al., "Role of gender in outcome after traumatic brain injury and therapeutic effect of erythropoietin in mice," Brain Research, vol. 1185, no. 1, pp. 301312, 2007.

[41] Y. Xiong, L. Dunyue, Q. Changsheng et al., "Effects of erythropoietin on reducing brain damage and improving functional outcome after traumatic brain injury in mice: Laboratory investigation," Journal of Neurosurgery, vol. 109, no. 3, pp. 510$521,2008$.

[42] Y. Zhang, M. Chopp, A. Mahmood, Y. Meng, C. Qu, and Y. Xiong, "Impact of inhibition of erythropoietin treatmentmediated neurogenesis in the dentate gyrus of the hippocampus on restoration of spatial learning after traumatic brain injury," Experimental Neurology, vol. 235, no. 1, pp. 336-344, 2012.

[43] J. J. Deeks, D. G. Altman, and M. J. Bradburn, "Statistical methods for examining heterogeneity and combining results from several studies in meta-analysis," in Systematic Reviews in Health Care: Meta-Analysis in Context, pp. 285-312, BMJ, 2001.

[44] K. Ker, P. Perel, and K. Blackhall, "Beta-2 receptor antagonists for traumatic brain injury: A systematic review of controlled trials in animal models," CNS Neuroscience and Therapeutics, vol. 15, no. 1, pp. 52-64, 2009.

[45] C. L. Gibson, L. J. Gray, P. M. W. Bath, and S. P. Murphy, "Progesterone for the treatment of experimental brain injury; a systematic review," Brain, vol. 131, no. 2, pp. 318-328, 2008.

[46] H. K. Baryan, S. M. Allan, A. Vail, and C. J. Smith, "Systematic review and meta-analysis of the efficacy of statins in experimental stroke," International Journal of Stroke, vol. 7, no. 2, pp. 150156, 2012.

[47] J. M. Wardlaw, C. P. Warlow, P. A. Sandercock, M. S. Dennis, and R. I. Lindley, "Neuroprotection disappointment yet aGAIN," The Lancet, vol. 356, no. 9229, article 597, 2000.

[48] W. Peng, J. Yang, Y. Wang et al., "Systematic review and meta-analysis of randomized controlled trials of Xingnaojing treatment for stroke," vol. 2014, Article ID 210851, 9 pages, 2014.

[49] J. A. C. Sterne, H. J. Newton, and N. J. Cox, Meta-Analysis in Stata: An Updated Collection from the Stata Journal, StataCorp LP, 2009.

[50] P. Perel, I. Roberts, E. Sena et al., "Comparison of treatment effects between animal experiments and clinical trials: systematic review," British Medical Journal, vol. 334, no. 7586, pp. 197200, 2007.
[51] P. Wheaton, J. L. Mathias, and R. Vink, "Impact of pharmacological treatments on outcome in adult rodents after traumatic brain injury: a meta-analysis," Journal of Psychopharmacology, vol. 25, no. 12, pp. 1581-1599, 2011.

[52] STAIR, "Recommendations for standards regarding preclinical neuroprotective and restorative drug development," Stroke, vol. 30, no. 12 , pp. 2752-2758, 1999.

[53] C. A. O'Connor, I. Cernak, and R. Vink, "Interaction between anesthesia, gender, and functional outcome task following diffuse traumatic brain injury in rats," Journal of Neurotrauma, vol. 20, no. 6, pp. 533-541, 2003.

[54] Y. Xiong, A. Mahmood, and M. Chopp, "Animal models of traumatic brain injury," Nature Reviews Neuroscience, vol. 14, no. 2, pp. 128-142, 2013.

[55] O. Danos, K. Davies, P. Lehn, and R. Mulligan, "The ARRIVE guidelines, a welcome improvement to standards for reporting animal research," Journal of Gene Medicine, vol. 12, no. 7, pp. 559-560, 2010.

[56] C. Kilkenny, W. J. Browne, I. C. Cuthill, M. Emerson, and D. G. Altman, "Improving bioscience research reporting: the arrive guidelines for reporting animal research," PLoS Biology, vol. 8, no. 6, Article ID e1000412, 2010. 

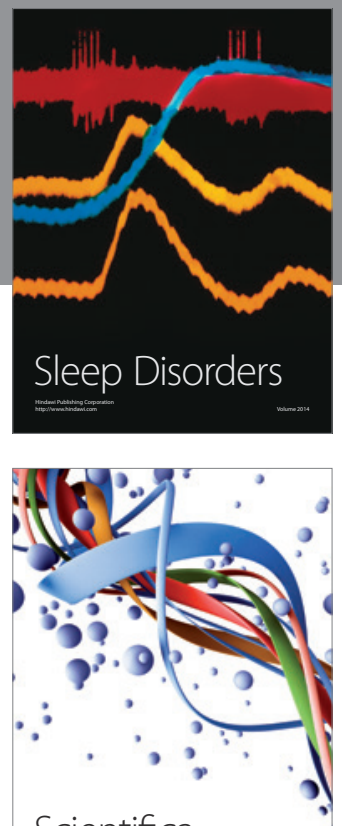

Scientifica
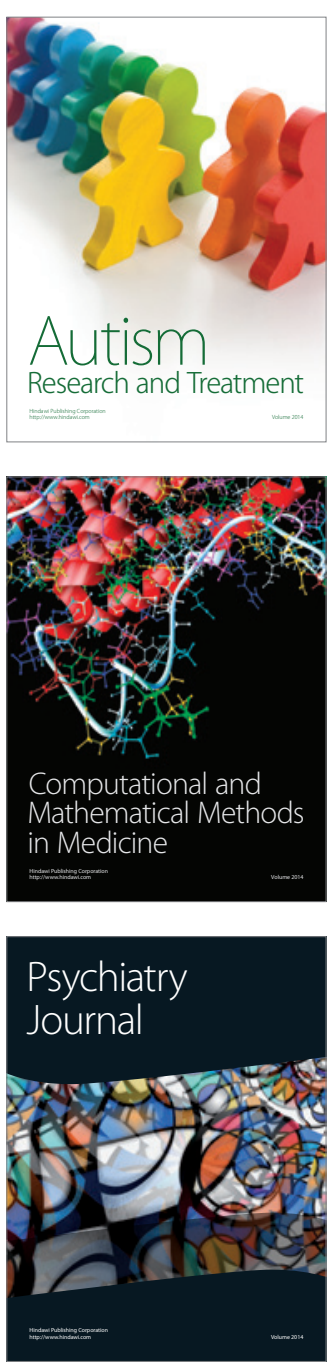
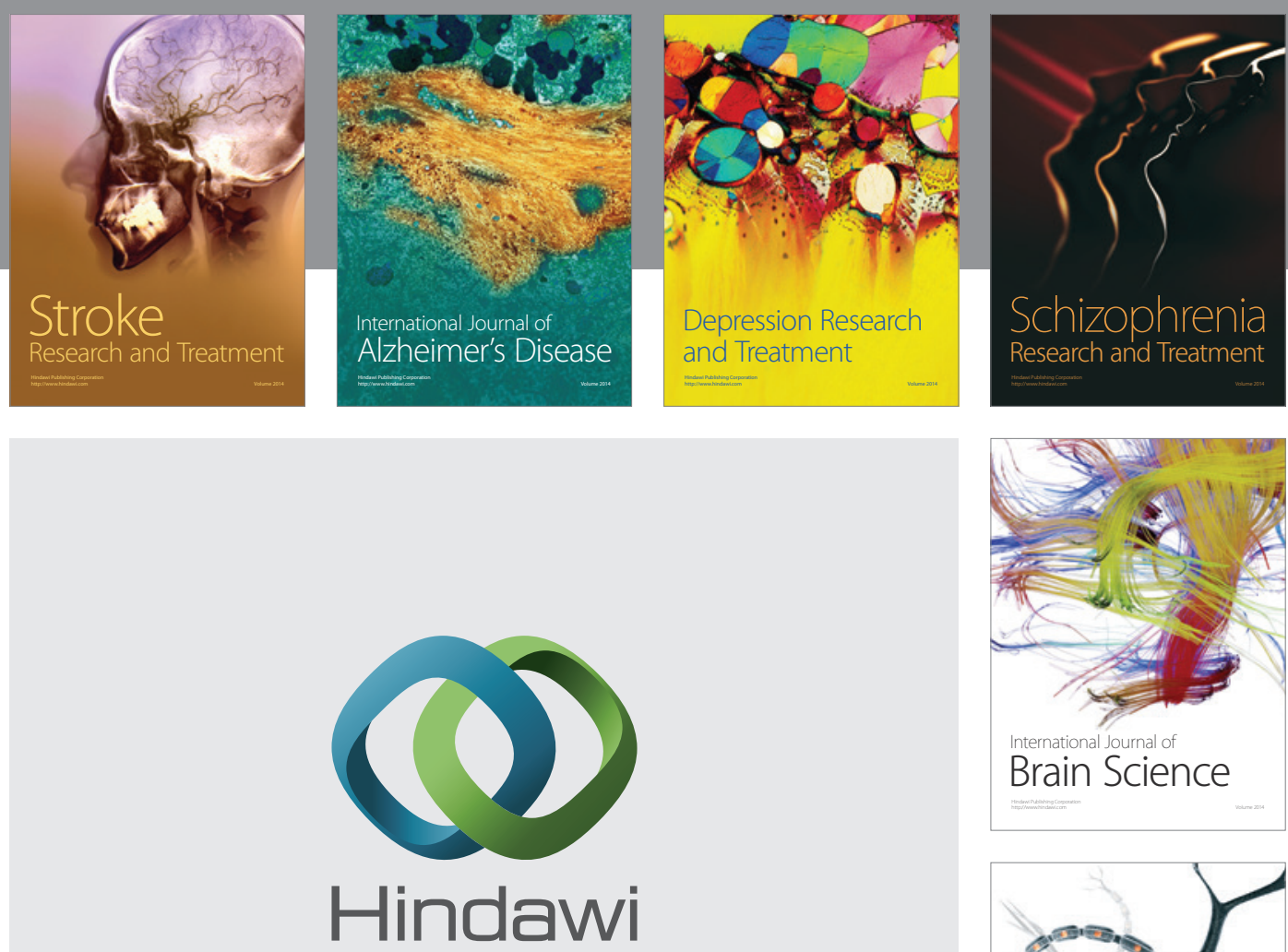

Submit your manuscripts at

http://www.hindawi.com
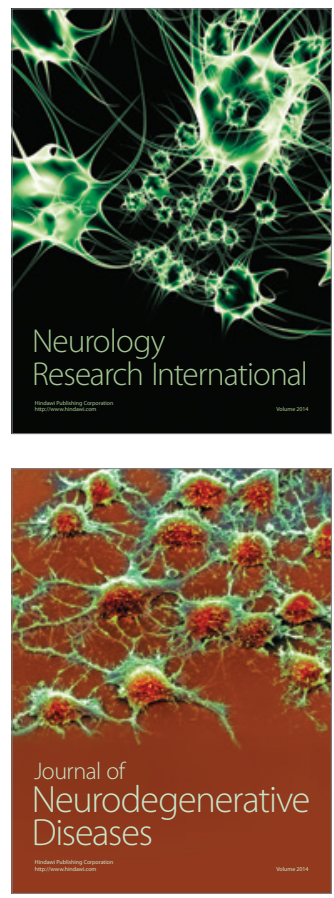

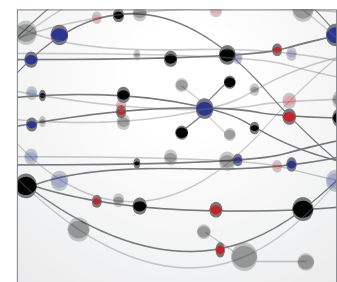

The Scientific World Journal
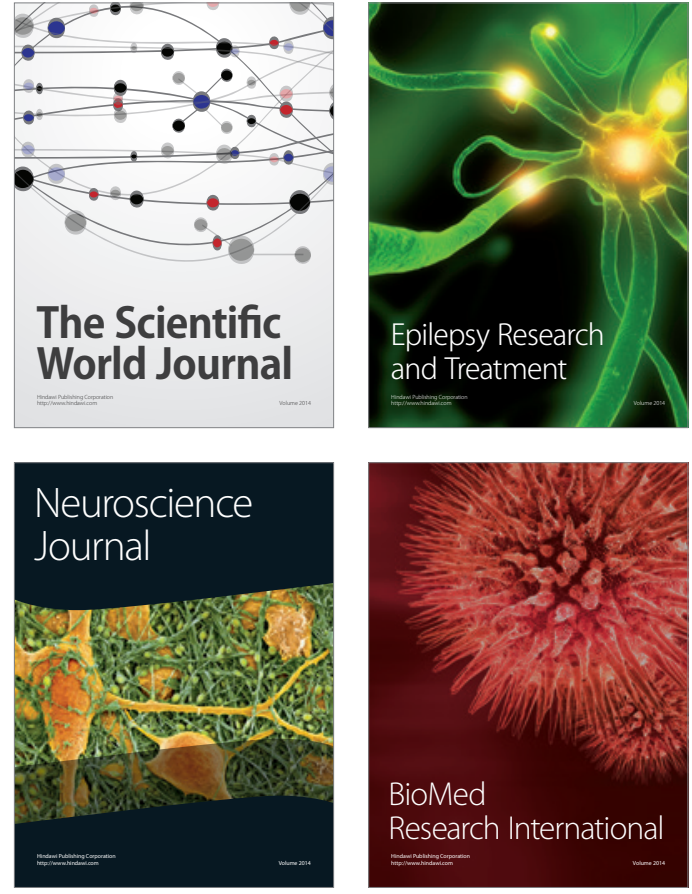

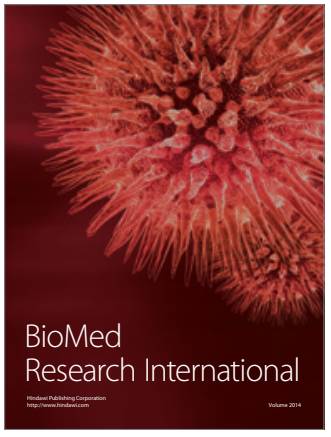

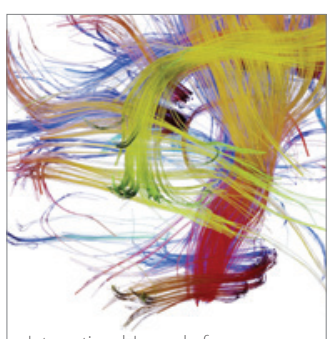

Brain Science

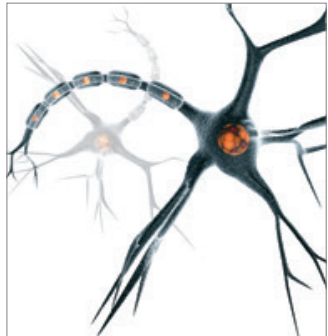

Neural Plasticity
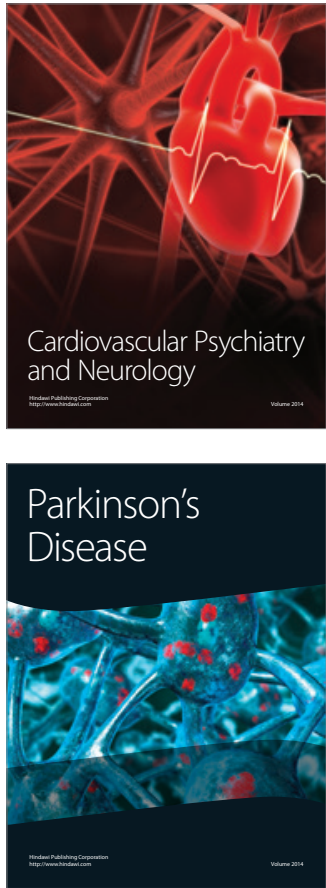